PROCEEDINGS OF THE

AMERICAN MATHEMATICAL SOCIETY

Volume 133, Number 3, Pages 931-936

S 0002-9939(04)07586-0

Article electronically published on August 24, 2004

\title{
BORDISM GROUPS OF SPECIAL GENERIC MAPPINGS
}

\author{
RUSTAM SADYKOV
}

(Communicated by Paul Goerss)

\begin{abstract}
The Pontrjagin-Thom construction expresses a relation between the oriented bordism groups of framed immersions $M^{m} \rightarrow \mathbb{R}^{n}, m<n$, and the stable homotopy groups of spheres. We apply the Pontrjagin-Thom construction to the oriented bordism groups $\mathcal{M}_{m, n}$ of mappings $M^{m} \rightarrow \mathbb{R}^{n}, m>n$, with mildest singularities. Recently, O. Saeki showed that for $m \geq 6$, the group $\mathcal{M}_{m, 1}$ is isomorphic to the group of smooth structures on the sphere of dimension $m$. Generalizing, we prove that $\mathcal{M}_{m, n}$ is isomorphic to the $n$-th stable homotopy group $\pi_{n}^{s t}\left(\mathrm{BSDiff}_{r}, \mathrm{BSO}_{r+1}\right), r=m-n$, where $\operatorname{SDiff}_{r}$ is the group of oriented auto-diffeomorphisms of the sphere $S^{r}$ and $\mathrm{SO}_{r+1}$ is the group of rotations of $S^{r}$.
\end{abstract}

\section{INTRODUCTION}

A point $x$ of a smooth mapping $f: M^{m} \rightarrow \mathbb{R}^{n}, m \geq n$, of a manifold is called regular if the rank of the differential $d_{x} f$ is maximal, i.e., equals $n$. Otherwise $x \in M^{m}$ is called singular. We say that a point $x$ is a definite fold singular point if in some coordinate neighborhoods about $x$ and $f(x)$ the mapping $f$ has the form

$$
f\left(t_{1}, \ldots, t_{n-1}, x_{n}, \ldots, x_{m}\right)=\left(t_{1}, \ldots, t_{n-1}, x_{n}^{2}+\cdots+x_{m}^{2}\right) .
$$

In this paper we study special generic mappings, which are mappings with only regular and definite fold singular points.

Suppose $f_{i}: M_{i}^{m} \rightarrow \mathbb{R}^{n}, i=0,1$, are two special generic mappings of closed oriented manifolds and there exists a special generic mapping $F: M^{m+1} \rightarrow \mathbb{R}^{n} \times$ $[0,1]$ of an oriented manifold $M^{m+1}$ with boundary $\partial M^{m+1}=M_{0}^{m} \cup\left(-M_{1}^{m}\right)$ such that

$$
F \mid M_{i}=f_{i}: M_{i}^{m} \rightarrow \mathbb{R}^{n} \times\{i\}, \quad i=0,1 .
$$

Then we say that the special generic mappings $f_{0}$ and $f_{1}$ are bordant. The classes of bordant mappings constitute an abelian group $\mathcal{M}_{m, n}$ under the operation of taking a disjoint union of mappings. The identity element of this group is represented by the mapping of the empty manifold. The inverse $-[f]$ to the bordism class of a mapping $f: M^{m} \rightarrow \mathbb{R}^{n}$ is represented by the composition of $f$ and a reflection of $\mathbb{R}^{n}$.

The bordism groups $\mathcal{M}_{m, n}$ were introduced by O. Saeki in [7] (see Problem 5.6). It turns out that for $m \geq 6$, the group $\mathcal{M}_{m, 1}$ is isomorphic to the $h$-cobordism group of oriented homotopy $m$-spheres $\Theta_{m}$ [8]. The restriction on the dimension $m$

Received by the editors August 14, 2003 and, in revised form, November 10, 2003.

2000 Mathematics Subject Classification. Primary 55N22; Secondary 55P42, 57R45.

Key words and phrases. Pontrjagin-Thom construction, special generic mappings, bordisms.

(C)2004 American Mathematical Society 
appears as a consequence of the same restriction in the Cerf pseudo-isomorphism theorem [1]. In view of the isomorphism $\Theta_{m} \approx \pi_{0}\left(\mathrm{SDiff}_{m-1}\right), m \geq 6$, where $\operatorname{SDiff}_{m-1}$ is the group of orientation-preserving auto-diffeomorphisms of the sphere $S^{m-1}$ [1], the Saeki theorem asserts that $\mathcal{M}_{m, 1} \approx \pi_{0}\left(\right.$ SDiff $\left._{m-1}\right)$ provided that $m \geq 6$.

For greater values of $n$, we will show that the group $\mathcal{M}_{m, n}$ is closely related to the space $\mathrm{SDiff}_{r} / \mathrm{SO}_{r+1}, r=m-n$, where $\mathrm{SO}_{r+1}$ is the space of rotations of $\mathbb{R}^{m+1}$ and the symbol '/' stands for the quotient of spaces. We recall that the $i$-th stable homotopy group of a topological space $X$ is defined as the direct limit of groups

$$
\pi_{i}^{s t}(X):=\lim _{N \rightarrow \infty} \pi_{i+N}\left(\Sigma^{N} X\right) .
$$

Here the space $\Sigma^{N} X$ is the $N$-th suspension of the space $X$. Let $\tilde{\pi}_{k}^{s t}(X)$ denote the $k$-th reduced stable homotopy group of $X$, i.e., the kernel of the homomorphism $\pi_{k}^{s t}(X) \rightarrow \pi_{k}^{s t}(p t)$ induced by the projection of $X$ onto a point $p t$. The $i$-th relative stable homotopy group $\pi_{i}^{s t}(X, Y)$ of a pair of topological spaces $(X, Y)$ is defined as $\tilde{\pi}_{i}^{s t}(X / Y)$. For a group $G$, let $B G$ be a space classifying principle $G$-bundles.

Theorem 1.1. There is a natural homomorphism $\mathcal{M}_{m, n} \rightarrow \pi_{n}^{s t}\left(\mathrm{BSDiff}_{r}, \mathrm{BSO}_{r+1}\right)$, $r \geq 0$, which is an isomorphism for $r>0$.

Corollary 1.2. The group $\mathcal{M}_{m, n}$ is isomorphic to $\tilde{\pi}_{n-1}^{s t}\left(\mathrm{SDiff}_{r} / \mathrm{SO}_{r+1}\right), r \geq 0$.

Proof. The long exact sequences of stable homotopy groups for pairs ( $\mathrm{SDiff}_{r}, \mathrm{SO}_{r+1}$ ) and $\left(\mathrm{BSDiff}_{r}, \mathrm{BSO}_{r+1}\right)$ fit into the commutative diagram

$$
\begin{aligned}
& \cdots \rightarrow \pi_{n}^{s t}\left(\mathrm{BSDiff}_{r}\right) \rightarrow \pi_{n}^{s t}\left(\mathrm{BSDiff}_{r}, \mathrm{BSO}_{r+1}\right) \rightarrow \pi_{n-1}^{s t}\left(\mathrm{BSO}_{r+1}\right) \rightarrow \cdots \\
& \downarrow \approx \downarrow \downarrow \downarrow \\
& \cdots \rightarrow \pi_{n-1}^{s t}\left(\mathrm{SDiff}_{r}\right) \rightarrow \pi_{n-1}^{s t}\left(\mathrm{SDiff}_{r}, \mathrm{SO}_{r+1}\right) \rightarrow \pi_{n-2}^{s t}\left(\mathrm{SO}_{r+1}\right) \rightarrow \cdots
\end{aligned}
$$

with vertical isomorphisms given by transgressions of the universal bundles over $\mathrm{BSO}_{r+1}$ and BSDiff $r$. By the Five Lemma, the remaining vertical homomorphisms

$$
\pi_{n}^{s t}\left(\mathrm{BSDiff}_{r}, \mathrm{BSO}_{r+1}\right) \longrightarrow \pi_{n-1}^{s t}\left(\mathrm{SDiff}_{r}, \mathrm{SO}_{r+1}\right)
$$

of the diagram are isomorphisms. Hence $\mathcal{M}_{m, n} \approx \pi_{n-1}^{s t}\left(\mathrm{SDiff}_{r}, \mathrm{SO}_{r+1}\right)$.

Corollary 1.3. The isomorphism $\mathcal{M}_{m, 1} \approx \pi_{0}\left(\operatorname{SDiff}_{m-1}\right)$ holds for all $m>0$.

Proof. For $n=1$, Theorem 1.1 asserts that $\mathcal{M}_{m, n}$ is isomorphic to the group

$$
\pi_{1}^{s t}\left(\mathrm{BSDiff}_{m-1}, \mathrm{BSO}_{m}\right) \approx \tilde{\pi}_{1}^{s t}\left(\mathrm{BSDiff}_{m-1} / \mathrm{BSO}_{m}\right)
$$

Let us prove that

$$
\tilde{\pi}_{1}^{s t}\left(\mathrm{BSDiff}_{m-1} / \mathrm{BSO}_{m}\right) \approx \pi_{0}\left(\mathrm{SDiff}_{m-1}\right) .
$$

The reduced Atiyah-Hirzebruch spectral sequence with coefficients in $\pi_{*}^{s t}(p t)$ for the space $X=\mathrm{BSDiff}_{m-1} / \mathrm{BSO}_{m}$ has the second term $\tilde{E}_{s, t}^{2}=\tilde{H}_{s}\left(X ; \pi_{t}^{s t}(p t)\right)$ and converges to $\tilde{\pi}_{s+t}(X)$. From $\tilde{E}_{0,1}^{2}=0$ and $\tilde{E}_{1,0}^{2}=\tilde{H}_{1}\left(X ; \pi_{0}^{s t}(p t)\right)$ we deduce that $\tilde{\pi}_{1}^{s t}(X)=\tilde{H}_{1}\left(X ; \pi_{0}^{s t}(p t)\right)=H_{1}(X ; \mathbb{Z})$. There is a commutative diagram

$$
\begin{aligned}
H_{1}\left(\operatorname{BSDiff}_{m-1}\right) & \approx H_{1}\left(\operatorname{BSDiff}_{m-1}, \operatorname{BSO}_{m}\right) \\
\pi_{1}\left(\operatorname{BSDiff}_{m-1}\right) & \approx \pi_{1}\left(\operatorname{BSDiff}_{m-1}, \mathrm{BSO}_{m}\right),
\end{aligned}
$$


where the horizontal isomorphisms are parts of the homology and homotopy long exact sequences for the pair $\left(\mathrm{BSDiff}_{m-1}, \mathrm{BSO}_{m}\right)$ and the vertical maps are Hurewicz homomorphisms. The group

$$
\pi_{1}\left(\text { BSDiff }_{m-1}\right) \approx \pi_{0}\left(\operatorname{SDiff}_{m-1}\right)
$$

is abelian. Therefore all the groups of the diagram are isomorphic and $\tilde{\pi}_{1}^{s t}(X) \approx$ $\pi_{0}\left(\mathrm{SDiff}_{m-1}\right)$.

Remark 1.4. For a definition of un-oriented bordism groups and generalizations of the group $\mathcal{M}_{m, n}$ we refer the reader to [7]. In [8] it is proved that for $m \geq 6$, the un-oriented bordism group of special generic functions is isomorphic to $\Theta_{n} \otimes$ $\mathbb{Z}_{2}$. The reasoning in the proof of Theorem 1.1 can be modified to show that in general the un-oriented bordism group of mappings of $m$-manifolds into $\mathbb{R}^{n}$ is isomorphic to $\pi_{n}^{s t}\left(\mathrm{BDiff}_{r}, \mathrm{BO}_{r+1}\right), r=m-n>0$, where Diff $r$ is the group of auto-diffeomorphisms of $S^{r-1}$ and $\mathrm{O}_{r+1}$ is the group of orthogonal transformations of $\mathbb{R}^{r+1}$.

Remark 1.5. For $r=0$, the homomorphism of Theorem 1.1 may not be an isomorphism. The group $\pi_{n}^{s t}\left(\mathrm{BSDiff}_{0}, \mathrm{BSO}_{1}\right)$ is trivial for all $n$. On the other hand, every homotopy sphere admits a special generic mapping and if the homotopy sphere does not bound a compact parallelizable manifold, then the special generic mapping represents a non-trivial element in $\mathcal{M}_{n, n}$ [7].

Remark 1.6. By [7, (5.5.7)], the groups $\mathcal{M}_{m, n}$ are trivial for $m-n=1,2,3$. We can deduce this fact from Theorem 1.1] Indeed, by 2] and [10, the natural inclusion $\mathrm{SO}_{r+1} \rightarrow \mathrm{SDiff}_{r}$ is a homotopy equivalence for $r=2,3$. Therefore $\pi_{n}^{s t}\left(\mathrm{BSDiff}_{r}, \mathrm{BSO}_{r+1}\right)=0$ for $r=1,2,3$.

The author would like to thank Y. B. Rudyak, A. N. Dranishnikov and S. Melikhov for helpful discussions.

\section{Proof of Theorem 1.1}

Let $f: M^{m} \rightarrow N^{n}$ be a smooth mapping of manifolds. We say that two points $x_{1}$ and $x_{2}$ of the domain are Stein equivalent if for some point $y \in N^{n}$ the points $x_{1}$ and $x_{2}$ belong to the same path component of the inverse image $f^{-1}(y)$. Identification of the Stein equivalent points leads to a space $W=W_{f}$ called the Stein factorization complex associated with the mapping $f$. If the space $W$ is endowed with the maximal topology with respect to which the factorization mapping $q_{f}: M^{m} \rightarrow W$ is continuous, then the mapping $f$ factors into the composition of $q_{f}$ and some continuous mapping $i_{f}: W \rightarrow N^{n}$.

In the case where $f$ is a special generic mapping, the Stein factorization complex has some especially nice properties, which are discussed in detail in [6]. Here we recall some of the properties without proofs.

- The topological space $W$ is a manifold with boundary. The dimension of $W$ is $n$.

- The manifold $W$ has a differentiable structure with respect to which the mappings $q_{f}$ and $i_{f}$ are smooth.

- The boundary of $W$ is diffeomorphic to the submanifold $S(f) \subset M$ of singular points of $f$, and the diffeomorphism is given by the restriction $q_{f} \mid S(f)$. 
- The mapping $i_{f}: W \rightarrow \mathbb{R}^{n}$ is an immersion.

- The manifold $W$ is parallelizable.

Let $\stackrel{\circ}{W}$ denote the complement in $W$ to an open collar neighborhood of the boundary $\partial W$. Then $\stackrel{\circ}{W}$ is a smooth manifold (with boundary) diffeomorphic to $W$. The restriction of the mapping $q_{f}$ to the inverse image $q_{f}^{-1}(\stackrel{\circ}{W})$ is a bundle with fiber diffeomorphic to the standard sphere [6]. We will denote the resulting sphere bundle by $\xi=\xi_{f}$.

Remark 2.1. There exist two mappings $f$ and $g$ of non-diffeomorphic manifolds into $\mathbb{R}^{n}$ that lead to equivalent fiber bundles $\xi_{f}=\xi_{g}$. Indeed, let $\Sigma_{f}$ and $\Sigma_{g}$ be two different 7-dimensional homotopy spheres and $f: \Sigma_{f} \rightarrow \mathbb{R}, g: \Sigma_{g} \rightarrow \mathbb{R}$ be two Morse functions with two critical points each. Then $f$ and $g$ are special generic, the Stein factorization complexes $W_{f}, W_{g}$ are segments and the bundles $\xi_{f}, \xi_{g}$ are trivial sphere bundles over subsegments $\stackrel{\circ}{W}_{f}$ and $\stackrel{\circ}{W}_{g}$ respectively. In particular, $\xi_{f}$ and $\xi_{g}$ are equivalent. We also indicate that the result of Saeki [8] implies that two special generic functions on different homotopy spheres are not bordant.

Let $B$ denote the closure of a collar neighborhood of the boundary $\partial W$ in the manifold $W$. The inverse image $q_{f}^{-1}(B)$ can be identified with the total space of the normal bundle of $S(f)$ in $M$. Since the $n$-manifold $W$ admits an immersion into $\mathbb{R}^{n}$, we have that $W$ is orientable and the singular manifold $S(f)$, being diffeomorphic to the boundary $\partial W$, is orientable as well.

The boundary of $q_{f}^{-1}(B)$ coincides with the set of points $q_{f}^{-1}(\partial B) \backslash S(f)$. We say that a bundle is linear if its structure group is a group of orthogonal transformations. Since the structure group of the normal bundle of $S(f)$ reduces to the group of rotations $\mathrm{SO}_{m-n+1}$, we obtain

Lemma 2.2 (O. Saeki, [6] Proposition 2.1]). The quotient mapping $q_{f}$ restricted to $q_{f}^{-1}(\partial B) \backslash S(f)$ is an orientable linear bundle.

In general the sphere bundle $\xi$ over $\stackrel{\circ}{W}$ has a structure group different from the orthogonal group. Let BSDiff $r$ be the space classifying smooth orientable bundles with fiber $r$-dimensional sphere (see construction in [5]) and $\mathrm{BSO}_{r+1}$ be the classifying space of $(r+1)$-dimensional orientable vector bundles. Then $\mathrm{BSO}_{r+1}$ is contained in the space BSDiff $_{r}$. Since the bundle $\xi$ restricted to the boundary $\partial \stackrel{\circ}{W}$ has orthogonal structure group, the mapping classifying the bundle $\xi$ over $\stackrel{\circ}{W}$ takes the pair $(\stackrel{\circ}{W}, \partial \stackrel{\circ}{W})$ into the pair $\left(\mathrm{BSDiff}_{r}, \mathrm{BSO}_{r+1}\right)$. To motivate the subsequent definition of a relative group $\mathcal{P}_{n}(X, Y)$, we observe that the manifold $\stackrel{\circ}{W}$ carries a framing $\tau$ of the stable tangent bundle defined by the immersion $i_{f}: \stackrel{\circ}{W} \rightarrow \mathbb{R}^{n}$.

Let $Y \subset X$ be a subset of an arcwise connected topological space $X$. By definition, an element of $\mathcal{P}_{n}(X, Y)$ is represented by a mapping $f:(N, \partial N ; \tau) \rightarrow(X, Y)$ of an $n$-dimensional manifold $N$ with a framing $\tau$ of the stable tangent bundle. Two mappings $f_{i}:\left(N_{i}, \partial N_{i} ; \tau_{i}\right) \rightarrow(X, Y), i=0,1$, represent the same element in $\mathcal{P}_{n}(X, Y)$ if and only if there is a mapping $f:\left(N, \partial N \backslash\left(N_{0} \cup N_{1}\right) ; \tau\right) \rightarrow(X, Y)$ such that

(1) $N_{0} \cup N_{1}$ is a submanifold of $\partial N$, 
(2) $f\left(\partial N \backslash\left(N_{0} \cup N_{1}\right)\right) \subset Y$,

(3) $f \mid N_{i}=f_{i}$ for $i=0,1$,

(4) the framing $\tau_{0}$ (respectively $\tau_{1}$ ) followed by the inward (respectively outward) vector normal to $T N_{0}$ (respectively $T N_{1}$ ) in $T N$ coincides with the framing $\tau \mid N_{0}$ (respectively $\tau \mid N_{1}$ ).

In terms of representatives the operation in $\mathcal{P}_{n}(X, Y)$ is given by taking the disjoint union of mappings.

We recall that $X$ is assumed arcwise connected.

Lemma 2.3. For each element of $\mathcal{P}_{n}(X, Y)$ there is a representative given by a mapping of a connected manifold with non-empty boundary.

Every special generic mapping $f: M^{m} \rightarrow \mathbb{R}^{n}$ gives rise to a manifold $\stackrel{\circ}{W}$ with stable tangent framing $\tau$ and a mapping

$$
\theta(f):(\stackrel{\circ}{W}, \partial \stackrel{\circ}{W} ; \tau) \rightarrow\left(\operatorname{BSDiff}_{m-n}, \mathrm{BSO}_{m-n+1}\right)
$$

representing some element $[\theta(f)]$ in $\mathcal{P}_{n}\left(\mathrm{BSDiff}_{m-n}, \mathrm{BSO}_{m-n+1}\right)$. Special generic mappings that represent the same element in $\mathcal{M}_{m, n}$ lead to the same class in $\mathcal{P}_{n}\left(\mathrm{BSDiff}_{m-n}, \mathrm{BSO}_{m-n+1}\right)$. Indeed, suppose $F$ is a bordism between special generic mappings $f_{0}$ and $f_{1}$. Then, being special generic, $F$ determines a classifying mapping $\theta(F)$ that provides a framed bordism between $\theta\left(f_{0}\right)$ and $\theta\left(f_{1}\right)$. Hence $\left[\theta\left(f_{0}\right)\right]=\left[\theta\left(f_{1}\right)\right]$. Since the group operation in $\mathcal{M}_{m, n}$ agrees with that in $\mathcal{P}_{n}\left(\right.$ BSDiff $\left._{m-n}, \mathrm{BSO}_{m-n+1}\right)$, we obtain a homomorphism

$$
h: \mathcal{M}_{m, n} \rightarrow \mathcal{P}_{n}\left(\text { BSDiff }_{m-n}, \mathrm{BSO}_{m-n+1}\right) .
$$

Let us find the kernel of the homomorphism $h$. If a mapping $f$ represents an element of the kernel, then, changing $f$ by bordism, we may assume that $\theta(f)$ sends $\stackrel{\circ}{W}_{f}$ into $\mathrm{BSO}_{m-n+1} \subset \mathrm{BSDiff}_{m-n}$. In [7] (see 5.5.6) O. Saeki showed that if $m>n$ and the bundle over $\stackrel{\circ}{W}_{f}$ determined by a mapping $f$ is linear, then $[f]=0 \in \mathcal{M}_{m, n}$. Thus, for $m>n$, the kernel of $h$ is trivial.

Lemma 2.4. The homomorphism $h: \mathcal{M}_{m, n} \rightarrow \mathcal{P}_{n}\left(\mathrm{BSDiff}_{m-n}, \mathrm{BSO}_{m-n+1}\right)$ is an isomorphism.

Proof. The homomorphism $h$ is injective. To show that $h$ is surjective, pick an arbitrary element in $\mathcal{P}_{n}\left(\mathrm{BSDiff}_{m-n}, \mathrm{BSO}_{m-n+1}\right)$. By Lemma 2.3, this element can be represented by some mapping

$$
\theta:(\stackrel{\circ}{W}, \partial \stackrel{\circ}{W} ; \tau) \rightarrow\left(\text { BSDiff }_{m-n}, \mathrm{BSO}_{m-n+1}\right)
$$

of a connected manifold $\stackrel{\circ}{W}$ with non-empty boundary. The mapping $\theta$ induces a sphere bundle $E$ over $\stackrel{\circ}{W}$ such that $\partial E$, which is the part of $E$ over $\partial \stackrel{\circ}{W}$, is the boundary of the total space of some linear disc bundle $D$ over $\partial \stackrel{\circ}{W}, \partial D=\partial E$. After smoothing the corners of $D \cup E$ we obtain a manifold $M$.

Since $\stackrel{\circ}{W}$ has a non-empty boundary, the framing $\tau$ of the stable tangent bundle $T \stackrel{\circ}{W} \oplus \varepsilon$ is the sum of a framing of $T \stackrel{\circ}{W}$ and the framing of the trivial 1-dimensional bundle $\varepsilon$. By the Smale-Hirsch theorem [3], the tangent framing of $T \stackrel{\circ}{W}$ determines a regular homotopy class of an immersion $i: \stackrel{\circ}{W} \rightarrow \mathbb{R}^{n}$. 
On the part $E$ of $M=E \cup D$ we define a regular mapping $f: E \rightarrow \mathbb{R}^{n}$ as the composition of the projection $E \rightarrow \stackrel{\circ}{W}$ and the immersion $i: \stackrel{\circ}{W} \rightarrow \mathbb{R}^{n}$. The mapping $f$ extends to a special generic mapping $f(\theta): M \rightarrow \mathbb{R}^{n}$ (see [6] Proposition 2.1]). The class of the mapping $f(\theta)$ in $\mathcal{M}_{m, n}$ maps under $h$ into $\theta$. Therefore $h$ is surjective.

By the Pontrjagin-Thom construction for manifolds with stable tangent framings, the group $\mathcal{P}_{n}\left(\mathrm{BSDiff}_{m-n}, \mathrm{BSO}_{m-n+1}\right)$ is isomorphic to the stable homotopy group $\pi_{n}^{s t}\left(\mathrm{BSDiff}_{m-n}, \mathrm{BSO}_{m-n+1}\right)$ (e.g., see [1]). This completes the proof of Theorem 1.1 .

\section{REFERENCES}

1. J. Cerf, La stratification naturelle des espaces de fonctions différentiables réelles et le théorème de la pseudo-isotopie, Publ. Math. Inst. Hautes Études Sci., 39 (1970), 5-173. MR 0292089 (45:1176)

2. A. E. Hatcher, A proof of the Smale conjecture, $\operatorname{Diff}\left(S^{3}\right) \simeq \mathrm{O}(4)$, Ann. of Math., 117 (1983), 553-607. MR 0701256 (85c:57008)

3. M. W. Hirsch, Immersions of manifolds, Trans. Amer. Math. Soc., 93 (1959), 242-276. MR $0119214(22: 9980)$

4. M. A. Kervaire, J. W. Milnor, Groups of homotopy spheres: I, Ann. of Math., 77 (1963), 504-537. MR 0148075 (26:5584)

5. S. P. Novikov, Differentiable sphere bundles, Amer. Math. Soc. Transl., 63 (1967), 217-244. MR 0174059 (30:4266)

6. O. Saeki, Topology of special generic maps of manifolds into Euclidean spaces, Topology Appl., 49 (1993), 265-293. MR 1208678 (93m:57031)

7. O. Saeki, Topology of special generic maps into $\mathbb{R}^{3}$, Mat. Contemp., 5 (1993), 161-186. MR 1305180 (95k:57037)

8. O. Saeki, Cobordism groups of special generic functions and groups of homotopy spheres, Japan. J. Math., 28 (2002), 287-297. MR 1947905 (2003k:57035)

9. P. Selick, Introduction to homotopy theory, Fields Institute Monographs, AMS, Providence, Rhode Island, 1997. MR 1450595 (98h:55001)

10. S. Smale, Diffeomorphisms of the 2-sphere, Proc. Amer. Math. Soc., 10 (1959), 621-626. MR 0112149 (22:3004)

11. R. E. Stong, Notes on cobordism theory, Princeton University Press and the University of Tokyo Press, Princeton, New Jersey, 1968. MR 0248858 (40:2108)

Department of Mathematics, University of Florida, Gainesville, Florida 32611

E-mail address: sadykov@math.ufl.edu 